\title{
THE RENAISSANCE OF EPISTEMOLOGY
}

\author{
LUCIANO FLORIDI
}

The renaissance of epistemology between the two world wars forms a bridge between early modern and contemporary philosophy of knowledge. At the turn of the century there had been a resurgence of interest in epistemology through an anti-metaphysical, naturalist, reaction against the nineteenth-century development of Neo-Kantian and Neo-Hegelian idealism. Within German-speaking philosophy, this reaction had its roots in Helmholtz's scientific reinterpretation of Kant, in Brentano's phenomenology, and in Mach's neutral monism. In British philosophy, it had acquired the specific nature of a rebuttal of Hegelianism by G. E. Moore and Bertrand Russell. And in America, the new pragmatist epistemology of William James and C. S. Peirce had directed attention away from the traditional a priori to the natural sciences. The interwar renaissance of epistemology, however, was not just a continuation of this emancipation from idealism. It was also prompted by major advances in mathematics, logic, and physics which engendered new methodological concerns (as in the influential tradition of French philosophers of science: Duhem, Poincaré, Bachelard). Hence among the traits that became prominent as a result of this renaissance, one may list an interest in mathematical, natural, and social sciences; criticism of the possibility of synthetic a priori truth; logical and semantic investigations which transformed epistemology from a theory of ideas and judgement into a theory of propositional attitudes, sentences, and meanings; a realist and naturalist orientation that tended to accommodate, if not to privilege, commonsensical and empiricist demands; a reconsideration of the role of philosophy as a critical exercise of analysis rather than as an autonomous and superior form of knowledge; and, finally, a disregard for the philosophy of history and the temporal dialectic of conceptual developments.

Of course, these perspectives never formed a unified programme of research. Philosophers between the wars took very different positions when developing their epistemological investigations, and the full reconstruction and analysis of these cannot be the task of this brief chapter. Its more limited aim will be 
to outline the discussions, within different traditions, of one central theme scepticism and the foundations of knowledge.

\section{THE TWO FACES OF SCEPTICISM}

Two questions set the issues for sceptical debates:

(K) Is knowledge possible?

(KK) Is epistemology possible?

(i.e. is an epistemology that answers $(\mathrm{K})$ possible?)

$(\mathrm{K})$ proceeds bottom-up whereas $(\mathrm{KK})$ works top-down in dealing with issues concerning the legitimacy of epistemic claims. At the beginning of our period, Edmund Husserl and the Neo-Thomistic philosopher Desire Mercier clarified further the normative character of these issues.

According to Husserl (I950), the justificatory ground of a theory of knowledge cannot be provided by other instances of knowledge. Following a Cartesian strategy, we can analyse potential instances of knowledge by affixing to them an 'index of questionability'. The sceptic questions the possibility of ever removing the index permanently, and Husserl argued that any supposed solution of the sceptical challenge simply by some extension of knowledge would be fallacious. The infringement of Husserl's principle can be considered of a piece with the 'naturalistic fallacy' in ethics: $(\mathrm{K})$ is not a question of natural science; by positing it, one asks whether what is considered to be knowledge justifiably deserves to be so described. Husserl formulated his principle by reacting against his previous psychologism and this explains why it can easily be formulated as an anti-naturalist dilemma: if a naturalised epistemology differentiates itself from a philosophical theory of knowledge by being empirically testable it raises the question of its own justification. Yet if it claims to be able to solve this foundational problem, it must provide a solution which cannot be in itself an instance of empirical knowledge, and with respect to which it must abandon its aspiration to gain the status of empirical science. The acceptance of this anti-naturalist stand was one of the reasons why Husserl argued for a purely conceptual approach to epistemology. Thus he held that the only correct way of dealing with $(\mathrm{K})$ is by means of principled and convincing arguments, by 'placing it in the logical space of reasons of justifying and being able to justify what one says' as Sellars was later to say (Sellars I963: I69).

But one can never be in a position to establish an answer to $(\mathrm{K})$ if no satisfactory reply to $(\mathrm{KK})$ can be provided. Questioning the possibility of epistemology rests on the problem of the criterion (Sextus Empiricus I976: II.2) which Mercier relaunched as 'le cheval du bataille du scepticisme' (Mercier I923; for a recent 
discussion, see Chisholm I973, I989). To know whether things really are as they seem to be, one needs a criterion for distinguishing between true and false appearances. But to know whether the criterion is reliable, one needs to know whether it succeeds in distinguishing between true and false appearances. And one cannot know this unless one already knows which appearances are true and which are false. So it seems that an epistemology cannot establish what, if anything, deserves to be called knowledge.

\section{ANTI-NATURALISM AND THE FOUNDATIONAL PROBLEM IN GERMAN-SPEAKING PHILOSOPHY}

Owing to his limited concern with scepticism, Kant had not considered (KK). The transcendental method of his first Critique was supposed to yield a justification for scientific knowledge, but what type of knowledge is exemplified by the Critique itself? How can it be justified without incurring a vicious circle? In I 807, the Kantian philosopher Jakob Friedrich Fries addressed this issue in his Neue oder anthropologische Kritik der Vernunft. Fries summarised the (KK) problem in terms of a trilemma: the premises of an epistemology can be dogmatically assumed, or justified by an endless chain of statements, or anchored to a psychological basis which is justificatory but not in need of a justification. During the I9Ios and the I920s there was a 'Fries-Renaissance', particularly in the work of Leonard Nelson (Haller 1974; Nelson I97I). Drawing on Fries's analysis, Nelson came to object to the entire project of an epistemology in the Cartesian, anti-sceptical, and justificatory sense, and to favour a more descriptive and psychologistic approach (Nelson I930, I965). Despite his criticism of foundational debates, Nelson's 'naturalised epistemology' contributed greatly to reawakening philosophers' interest in the foundational issue in the late I920s and early I930s. One philosopher especially influenced by his work was Moritz Schlick.

Schlick endorsed the Cartesian requirement that there be an absolutely certain foundation of knowledge. He also accepted that it was 'self-evident that the problem of the foundation of all knowledge is nothing else but the question of the criterion of truth' (Schlick I979: II, 374) and supported a correspondence theory of truth. However, in his view 'anyone who holds that the Cartesian thesis [i.e. the direct perception of a fact of consciousness] constitutes knowledge will inevitably be drawn into a similar circle [i.e. Fries's trilemma]' (Schlick I925 [1974: 86]). So he came to defend a foundationalism according to which there are objective facts, external to the knower's doxastic states, that are accessible by the knower and capable of justifying the knower's beliefs in a way that is sufficient for knowledge. 
According to Schlick, protocol propositions, that is, basic statements, 'in absolute simplicity, without any forming, change or addition, set forth the facts, whose elaboration constitutes the substance of all science' (Schlick 1979: II, 370, see also $400-I_{3}$ ). But although they are logically prior to other factual propositions, they are themselves grounded on a prior layer of indubitable, empirically contentful, 'affirmations' (Konstatierungen). These affirmations, for example, 'there is red here now', are expressions of immediate, simple, existential experiences which provide a genuine acquaintance with reality even though they are wholly subjective, fleeting, and ineffable. They are indubitable because to understand their meaning is to understand their truth; hence they constitute the basic form of evidence for protocol propositions, which are constantly tested against them. These 'affirmations' are, therefore, the foundation of science, not in a fixed, static, sense, but only in the sense that they form the constantly renewed ground necessary and sufficient for its open-ended development.

Schlick's foundationalism requires a verificationist semantics, an ostensive theory of meaning of elementary propositions, and a conception of ineffable experience of the world which is immediately 'given' to the subject. As a result it appeared to critics such as Otto Neurath similar to a philosophy of intuition; they argued that it was beset with solipsistic difficulties and contained unacceptable metaphysical theses.

Neurath's view was that only a nominalist and constructivist interpretation of the nature of protocol propositions - as actual statements, expressed in a physicalistic language, recording empirical observations - is metaphysically acceptable. Scientific theories could be grounded, empirically, on these basic protocol sentences, but no position 'external' to language is achievable. Sentences could be compared only with other sentences. Direct reference to external reality or internal experience is to be avoided as empty metaphysics. Since protocol sentences are intrinsically fallible and hence always corrigible in principle, any Cartesian demand for absolute certainty is unrealistic and misleading. For Neurath, the epistemic justification of science was not to be achieved by means of an appeal to external facts or alleged intuitions, but internally, through logical coherence (which did not necessarily exclude some ordering relations), instrumental economy, pragmatic considerations of social and scientific ends, a rational use of conventions by the scientific community, and a constantly open and public debate. Following Duhem, Neurath argued that, given an apparently successful theory, rival explanations can be made to fit the same evidence that supports it, and that in replacing or revising a theory, hypotheses and observation statements come under scrutiny as whole networks, not individually. Practical expedience rather than absolute truth was determinant. 
Neurath summarised his position in a famous analogy: 'We are like sailors who have to rebuild their ship [the system of knowledge] on the open sea, without ever being able to dismantle it in dry-dock and reconstruct it from its best components' (Neurath I983: 92). Hegel had stressed a similar point: 'the examination of knowledge can only be carried out by an act of knowledge. To examine this so-called instrument is the same thing as to know it. But to seek to know before we know [i.e. Kant's critical project] is as absurd as the wise resolution of Scholasticus, not to venture into the water until he had learned to swim' (Hegel I830 [I975: I4]). This was not the only similarity between Neurath's and Hegel's coherentism. A direct consequence of the former's holistic and non-subjectivist 'pan-internalism' was a strong tendency towards a unified and synoptic approach to the entire domain of human knowledge. This 'epistemological totalitarianism' was a feature shared by other approaches to the foundational problem, such as Hilbert's and Cassirer's, which were similarly suspicious of the Cartesian subjective turn, though favourable to Kantian constructionism rather than pure coherentism. In Neurath, this tendency was reflected in his project for an International Encyclopedia of Unified Science (1938-70). The programme of the Encyclopedia was presented as an explicit development of the ideas of the French Enlightenment, of Diderot's famous Encyclopédie. But the latter had a Cartesian basis, so the comparison should rather be with Hegel's Encyclopaedia of the Philosophical Sciences, although Neurath's project had a methodological justification in place of Hegel's ontological one.

Schlick was firmly hostile to Neurath's coherentism. If epistemic statements are not based on a specific set of more basic protocol propositions ultimately rooted in sense-experience of the world, all propositions may be regarded as in principle corrigible and their truth can only consist in their mutual agreement within the system of knowledge. Schick argued that such coherence provides too little - truth can be equated to logical consistency only in a formal system but not in an empirical science, since a coherent tale may otherwise become as acceptable as a scientific fact. Moreover, the absence of coherence leaves it utterly unclear what propositions may need to be revised, eliminated, or adjusted, and how. But despite Schlick's criticisms, Neurath's coherentism found an ally in the pragmatist movement, which was equally anti-Cartesian. Quine's fallibilist and holistic, naturalised epistemology can be interpreted as its latest development (Quine I969, I992).

Another philosopher deeply influenced by the 'Fries Renaissance' was Popper (Popper 1962, I979). His discussion of the problem of the nature of basic statements in scientific theory and his dynamic solution - we can simply leave the 
presuppositions of a specific science open to the possibility of further testing became the source of some later important works on the foundational problem (Albert 1978, I985; Apel I975; Lakatos 1978). It was also the origin of a significant modification in the interpretation of $(\mathrm{KK})$, now understood no longer as a meta-epistemological problem, but as a problem concerning the foundation of scientific knowledge.

\section{COHERENTISM, NATURALISM, AND THE REFUTATION OF SCEPTICISM IN BRITISH PHILOSOPHY}

Coherentism in epistemology is a natural ally of anti-realism in ontology, and both find a fertile environment in idealistic philosophies, whose claims about the contradictory nature of appearances in defence of a monistic supra-naturalism may easily make use of the sceptic's dualist anti-naturalism (Hegel I 802). Neurath had charged Schlick with metaphysical inclinations but found himself sharing a coherentist epistemology not entirely different from that of some idealists.

During the post-First World War period the most interesting and influential idealist epistemology remained that of F. H. Bradley (Bradley I9I4, I922, I930). Bradley held that the Absolute remains unknowable because knowledge is necessarily discursive and relational, and hence always inadequate with respect to the continuously unified nature of reality. Ideally, reality, its true description and its complete knowledge are identical. In practice, knowledge remains fragmentary and truth can only be more or less adequate. Knowledge, including science, is like the biography of a person: no matter how accurately written, it is still only partly true and conspicuously unsatisfactory when compared to actual life. Justification, understood as the process through which possible instances of partial knowledge may or may not be included in the epistemic system, is interpreted in terms of coherence. There is only one, ultimate, complete, accurate description of reality and every limited truth, insofar as it is true, concurs with every other limited truth in realising it.

The kind of holistic coherence involved here that Bradley and his friend Bernard Bosanquet (I920) seemed to have in mind, and that was further refined by J. J. Joachim (I939) and Brand Blanshard (I939), is richer than Neurath's mere logical consistency. It can be compared to the web of internal, reciprocal relations linking a set of words in a complex crossword, or a set of pieces in a puzzle: each truth is meant to interlock, meaningfully and uniquely, with the other components to constitute the whole, final system. It was justified by ontological considerations and some of the problems affecting later coherentist epistemologies, such as Neurath's, can be interpreted as stemming from the lack of a similar metaphysical commitment. 
The reaction against idealism amongst British philosophers had been motivated in part by scepticism concerning these metaphysical commitments (e.g. internal relations and organic wholes). Amongst these realist critics, G. E. Moore's positive approach to epistemology was distinctive and influential. Against the sceptical position, Moore held that there are many commonsensical beliefs that everyone is naturally inclined to hold and that are endorsed upon reflection by all. These beliefs have the highest (a) presumptive and (b) pervasive credibility. Their presumptive credibility means that it is virtually impossible to doubt them or dissent from them. We are perfectly entitled to start by accepting them as being prima facie epistemically justified, leaving to the sceptic the hard task of showing that, on the whole, there are better reasons to believe that not-p rather than $p$. Their pervasive credibility means that the sceptic's claims are incoherent. The denial of p's credibility presupposes $p$ itself or a system of credible ps.

Moore based his defence of common sense on (a) and (b) (Moore I925, I939). Re-evaluating Reid's philosophy, he concluded that there is an objective and mind-independent, physical reality, which we can come to know through our sense-data. However, how our immediate awareness of these sense-data is related to our knowledge of the world remained a constant problem for Moore and led to Ducasse's adverbial realism: sensations are ways of perceiving an object, not entities, so in looking at a tomato we are not directly aware of red sense-data but 'we are being appeared to redly by an object' (Ducasse I942).

Moore's antisceptical strategy rested on an inadequate, if influential, assessment of scepticism. Ancient sceptics themselves had already disposed of (b) (Sextus Empiricus I976: II, I44-203). Scepticism is not a doctrine but a process of immanent criticism that gradually rots away Neurath's ship from within. As for (a), it could not be employed against the sceptic without presupposing an antisceptical answer to $(\mathrm{K})$ and hence begging the question. Moore's reliance on common sense suggests an appeal to naturalised epistemology to provide an answer to $(\mathrm{K})$. But since $(\mathrm{K})$ involves a de jure epistemological problem this would have meant committing in epistemology that naturalistic fallacy which Moore himself had helped to clarify in ethics. Moore's anti-Cartesian naturalism could thus only be defended convincingly by conceding something to the sceptic, as Gassendi and Hume had done before, and Wittgenstein, in effect, did later (Wittgenstein 1969).

The problem of our 'knowledge of the external world' was also a central theme in Russell's writings. In response to this traditional problem, he developed the famous distinction between 'knowledge by acquaintance and knowledge by description' which is grounded upon his theory of descriptions and his 'fundamental epistemological principle' that 'any proposition which we can understand must be composed of constituents with which we are acquainted' 
(Russell I9I2: 9I). In The Problems of Philosophy (I9I2) Russell had argued that the sense-data, concerning colour, shape, texture, etc., with which we are acquainted in sense-experience give rise to self-evident, intuitive, truths which provide a foundation for our knowledge of the external world. This sounds like familiar empiricist foundationalism; but there remain questions concerning the ontological status of these sense-data and how truths concerning them relate to truths concerning physical objects such as a table, and Russell's answers to these questions were unusual. In his I9I 3 'Theory of Knowledge' (in Russell I984) he took it that sense-data are physical but located in private spaces, though once he adopted neutral monism in I92 I he regarded them as neutral, antecedent to the mental/physical distinction (Russell I92 I: lect. VIII). Physical objects themselves can only be 'known by description', and from I9I 3 he held that this knowledge rests upon a complex 'logical construction' whereby the different private spaces (or 'perspectives') in which sense-data are located are somehow combined into a public space so that truths about physical objects can be interpreted in terms of the contents of the perspectives which make them up.

This complex constructionism never gained much support and in later writings (e.g. Russell 1927) Russell developed alternatives to it. But what is more interesting about his writings from I92 I onwards is the way in which he began to develop an 'externalist' conception of knowledge. In The Problems of Philosophy (I9I2: ch. I3) Russell set out the classic analysis of knowledge as 'true, justified, belief' while observing in passing the difficulty that there are cases in which, because of some unfortunate circumstances, $\mathrm{S}$ may be fully justified in believing that $\mathrm{p}, \mathrm{p}$ may be true and yet $\mathrm{S}$ may still not really know that $\mathrm{p}$ because $\mathrm{S}$ has in fact merely hit on the truth by chance. Russell did not linger with this observation, which he thought he could handle by raising the standards for justification (when the point was famously revived by Edmund Gettier it gave rise to a branch of modern epistemology - see Gettier I963). But what happened from I92 I onwards, when Russell became converted to neutral monism and was profoundly influenced by behaviourist psychology, was that he began to develop and defend a conception of knowledge as an inner state which accurately, or reliably, represents some feature of the world (he sets out the now famous 'thermometer-analogy' for knowledge in Russell I92 I: I8I). Russell applied this first to memory; in later writings he extends its application furtherin particular using it to develop a sophisticated externalist account of induction in his last major work (I948). It should be added, however, that on this last matter Russell had been anticipated by F. P. Ramsey (I93 I: I97), who had also generalised Russell's tentative presentation of an externalist conception of knowledge into the bold statement that 'a belief [is] knowledge if it is (i) true, (ii) certain, (iii) obtained by a reliable process ... [that is, one that] must be caused by what 
are not beliefs in a way or with accompaniments that can be more or less relied on to give true beliefs' (Ramsey I93I: 258). The merits of this position are now widely appreciated, partly indeed because it is thought to provide a better solution to Russell's 'Gettier problem' than Russell had offered. Hence in this respect Russell and Ramsey together set the stage for a central dimension of contemporary epistemology.

\section{PRAGMATIST EPISTEMOLOGIES IN AMERICAN PHILOSOPHY}

The philosophers who best represent the epistemological renaissance during the interwar period in the United States are John Dewey and C. I. Lewis.

In Dewey, the influence of Darwinism, psychology, and Hegel's dialectics is reflected in an anti-Cartesian, naturalistic epistemology (Dewey 1925, 1929, 1938). He contended that traditional epistemology has been beset by four main errors:

I. foundationalism;

2. the assumed primacy of knowledge in our access to reality;

3. the artificial distinction between the known reality, the knowing process and knowledge as its outcome;

4. the spectator theory of knowledge - the conception of the object of knowledge as a detached reality, passively contemplated by an external and uninvolved spectator.

Consequently, he attempted to rectify these errors by arguing that:

I. knowledge is a non-hierarchical and relational phenomenon that emerges from natural and fallible interactions between two dependent relata, mind and nature ('contextualism');

2. non-reflective and non-cognitive experiences provide direct access to the world;

3. the epistemic continuum includes both the process of enquiry, as a series of selfcorrective, organising acts, and knowledge, defined as the attainment of 'warranted assertibility' and interpreted as its appropriate completion;

4. the object of knowledge is just the observable outcome of experimental procedures.

For Dewey, then, the elaboration of knowledge is the semiotic means - the process of inferential signification - through which we can facilitate the experimental determination of future consequences, by bridging the gap between the known and the unknown. Cognition is an always-contextualised way of dealing with a problem situation ('epistemological immanentism'). Rather than speaking of some static correspondence with reality, satisfactory theories or concepts are those that turn out to be usefully reliable in all the practical and theoretical endeavours for which they are developed. Dewey's critique of Cartesian epistemology has been more influential (as in the work of Quine, Putnam, and 
Rorty) than his instrumentalism has been. Russell and Reichenbach, for example, immediately objected that the latter failed to account for mathematical sciences.

C. I. Lewis stands in a similar relation to Kant as Dewey does to Hegel. Following Kant, he analysed knowledge in terms of judgements whose correctness depends on their relations to their references, and whose justification requires supportive reasons. Knowledge, he held, has a hierarchical structure, based on the infallibility of sensory states. The sensory given - the immediate apprehension of what is presented to the senses - is devoid of a conceptual structure and is not yet a form of knowledge, for there can be no possibility of error, since the apprehension of what is sensibly presented and the latter's existence qua appearance are indistinguishable, and, contrary to Descartes, Lewis held that we can speak of knowledge only if error is possible. But the epistemically infallible given supports 'terminating judgements', which are certain and have a predictive value. Their logical form is: 'Given the sensory cue S, if the possible action $\mathrm{A}$ is performed then the expected experiential appearance E will occur.' The certainty of terminating judgements is established by their being verified by practical tests concerning the predicted consequence. All other empirical judgements which concern objective facts different from the sensory given (e.g. physical objects) are non-terminating. Non-terminating judgements are at most probable and increasingly verifiable, never absolutely certain. Their logical form is ' $\mathrm{If} \mathrm{O}$ is a physical object, then if $\mathrm{S}$ and $\mathrm{A}$ occur, then in all probability E will occur.' They entail terminating judgements, on which they are based. Lewis argued that probability ultimately requires and is based on certainty, for if no judgement could count as certain even probable judgements would be impossible (they would be merely 'probably probable'). The grounding relation between terminating and non-terminating judgements grants that the former, if verified, confer on the latter their degree of probability and hence their meaningfulness.

Although for Lewis the a priori was coextensive with the analytic, a priori concepts, categories, logical relations and truths were not empirically irrelevant linguistic conventions. They provide the indispensable ordering, taxonomic, and interpretative criteria without which the given could not rise to the level of knowledge but would remain unintelligible. Thus they have the crucial role of conceptualising and giving sense to the unlimited and undetermined continuum of the sensory given. Contrary to Kant's view, a priori structures are creative constructions that do not constrain but need to be adequate to experience and hence are not fixed. Having a hermeneutic function, they represent stable semantic commitments which are modifiable or replaceable in the light of 
pragmatic requirements: intelligibility, order, simplicity, economy, comprehensiveness, resilience and, in the long run, intellectual and practical satisfaction.

Lewis tried to reconcile his 'phenomenalist' foundationalism with a realist theory of perception of physical objects (terms in non-terminating judgements denote genuine physical objects, not just phenomena or subjective appearances), without resorting to Kant's doctrine of the synthetic a priori, but his original 'pragmatic apriorism' remained a rather isolated attempt to develop a transcendental epistemology between the wars. 